\title{
A DISTRIBUTED-DELAY MODEL FOR THE LOCAL POPULATION DYNAMICS PARASITOID-HOST SYSTEM
}

\author{
Fred Adler \\ Center for Applied Mathematics \\ Cornell University \\ Lincoln Smith \\ Department of Entomology \\ Cornell University \\ Carlos Castillo-Chavez \\ Biometrics Unit \\ Department of Plant Breeding and Biometry \\ Cornell University
}

$\mathrm{BU}-1012-\mathrm{M}$

Revised January 1989

In: Mathematical approaches to resource management and epidemiology. Lecture Notes in Biomathematics. (C. Castillo-Chavez, S.A. Levin and C. Shoemaker, eds.) springer Verlag (in press). 


\begin{abstract}
A data-based mathematical model to assess the effectiveness of one species of parasitoid as a control agent to fly pupae is introduced and analyzed.

The analysis of this distributed-delay model provides us with several ideas regarding biological control. For example, a host-parasitoid model in which the recruitment of susceptible hosts is unaffected by local dynamics, has a tendency to converge to a stable equilibrium whether or not the parasitoids can persist. This equilibrium can have arbitrarily small numbers of susceptible hosts when the parasitoids are extraordinarily efficient. Finally, we show that the ability of parasitoids to persist can be established in terms of basic fly and parasitoid life history parameters.
\end{abstract}




\title{
A Distributed-Delay Model for the Local Population Dynamics of a Parasitoid-Host System
}

\author{
Fred Adler \\ Center for Applied Mathematics \\ Cornell University \\ Lincoln Smith \\ Department of Entomology \\ Comell University \\ Carlos Castillo-Chavez \\ Biometrics Unit \\ Department of Plant Breeding and Biometry \\ Cornell University
}

\section{INTRODUCTION}

In several instances, parasitoids have proven to be effective biological control agents of agricultural pests (Caltagirone, 1981; Huffaker and Messenger, 1976; Greathead, 1986). A number of models have been designed to indicate the stability properties of parasitoid-host systems under a variety of assumptions about the parasitoids (Hassell, 1986; Hassell and May, 1973; Murdoch et al., 1985). Our objective is the development of models that will be quantitatively predictive in a controlled environment in order to establish bounds on the effectiveness of this parasitoid over a range of environmental conditions. In this paper we develop a data-based model designed to assess the effectiveness of one species of parasitoid in a laboratory situation. We have therefore chosen to ignore the difficulties associated with measuring and modelling the spatial patterns of searching and foraging which may control the dynamics in the real world (Hassell, 1978; Reeve and Murdoch, 1985).

Smith and Rutz $(1985,1986,1987)$ have recently described the demographic statistics of the parasitoid Urolepis rufipes Ashmead (Hymenoptera: Pteromalidae) parasitizing Musca domestica L., the house fly, in New York State. To assess the possible value of this parasitoid for biological control, we have constructed a simple model for the dynamics of this parasitoid in a controlled environment. The parameters for this model have been measured at a variety of temperatures (Smith and Rutz, 1986), and analysis of the model with these values will be soon forthcoming. 
The life cycle of $U$. rufipes has been described by Smith and Rutz (1985). The following features have formed the basis of our model. First, in contrast to the systems studied by Hassell (1978), the summer breeding seasons of both flies and parasitoids are characterized by multiple overlapping generations. We have thus modelled using continuous rather than discrete time. Second, this particular parasitoid appears to avoid super-parasitism; adult parasitoids do not lay their eggs in fly pupae which have already been stung. Extensive super-parasitism is characteristic of many species, and could substantially complicate the dynamics. Finally, we assume that the flies disperse over a larger scale than that of the model, whereas local parasitoid densities respond only to local conditions (Danthanarayana, 1986). This formulation is thus most appropriate for modelling local sources, rather than local control, of adult flies which are usually considered the pest.

\section{Development of the Model}

The dynamics we model follows the flow pictured in Figure 1.

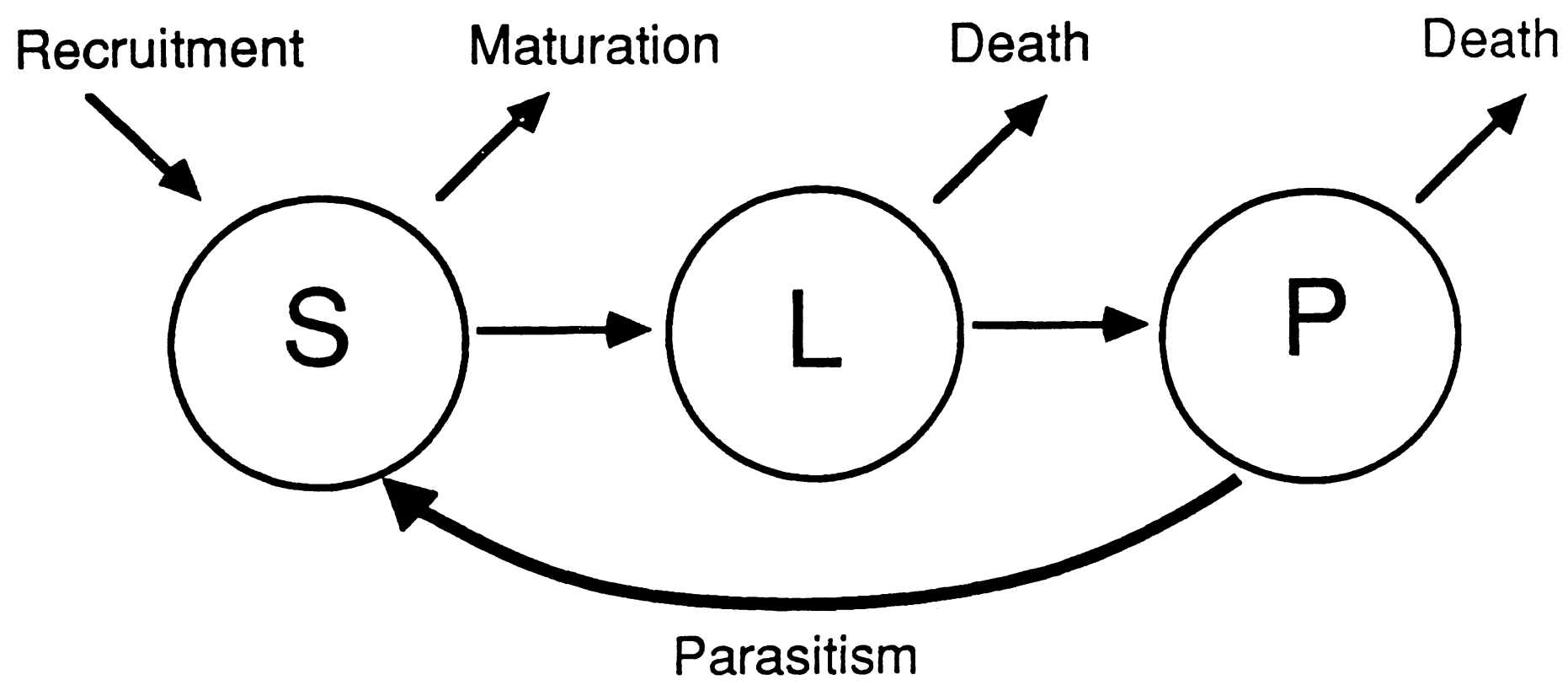

Figure 1. Diagram of the basic model. S - susceptible hosts, L - parasitized hosts (=parasitoid larvae), P - parasitoid adults.

Assuming an exponentially distributed development time for the parasitoid larvae and fly pupae, and ignoring age structure in the larval and adult parasitoids, seasonality in the life history parameters, and changes in the parasitoid sex ratio, we arrive at the following model: 


$$
\begin{gathered}
S^{\prime}=\beta-l P S-v S \\
L^{\prime}=l P S-m L-\delta L \\
P^{\prime}=m L-n P
\end{gathered}
$$

where ${ }^{\prime}=d / d t$, and the parameters are defined in Table 1

\begin{tabular}{|c|c|}
\hline Parameter & Meaning \\
\hline$\beta$ & Rate of addition of fly pupae from the environment \\
$l$ & Some measure of adult parasitoid fecundity \\
$v$ & Fly maturation rate \\
$m$ & Parasitoid larval maturation rate \\
$\delta$ & Parasitoid larval death rate \\
$n$ & Adult parasitoid death rate \\
\hline
\end{tabular}

This model, which follows closely the framework of Anderson and May (1981), can be made far more realistic for the system under consideration without becoming intractable, so analysis is postponed until the general model has been developed.

In reality, larval development time is very ill-matched by the exponential removal rate (Smith and Rutz, 1986). Following Murdoch et al (1987), we could utilize a discrete delay of the mean development time. However, analysis with a distributed delay describing the full structure of development times not only poses no more difficulties but is more realistic.

Some data on the development times of $U$. rufipes are available in Smith and Rutz (1986). We incorporate this into our model by introducing a density of development times $p(u)$. Figure 2 illustrates a typical form of such data. Denoting by $q$ the fraction of parasitoid larvae which complete development yet fail to emerge, we have

$$
\int_{0}^{\infty} p(u) d u=1-q
$$

$q$ can be quite large at high temperatures (Smith and Rutz, 1986). We observe that $\int_{0}^{\infty} p(u) e^{-\delta u} d u$ represents the expected fraction of eggs laid which will mature successfully into adult parasitoids. This fraction includes losses due both to larval death during development and to the failure of fully developed larvae to mature, and plays a critical role in determining the location and stability of equilibria in the system. The maturation term $m L$ appearing in equations (1b) and (1c) is then replaced by

$$
\int_{0}^{\infty} l P(t-u) S(t-u) p(u) e^{-\delta u} d u
$$


If $p(u)=m e^{-m u}$ this model reverts to model (1) under suitable initial conditions (see Appendix A).

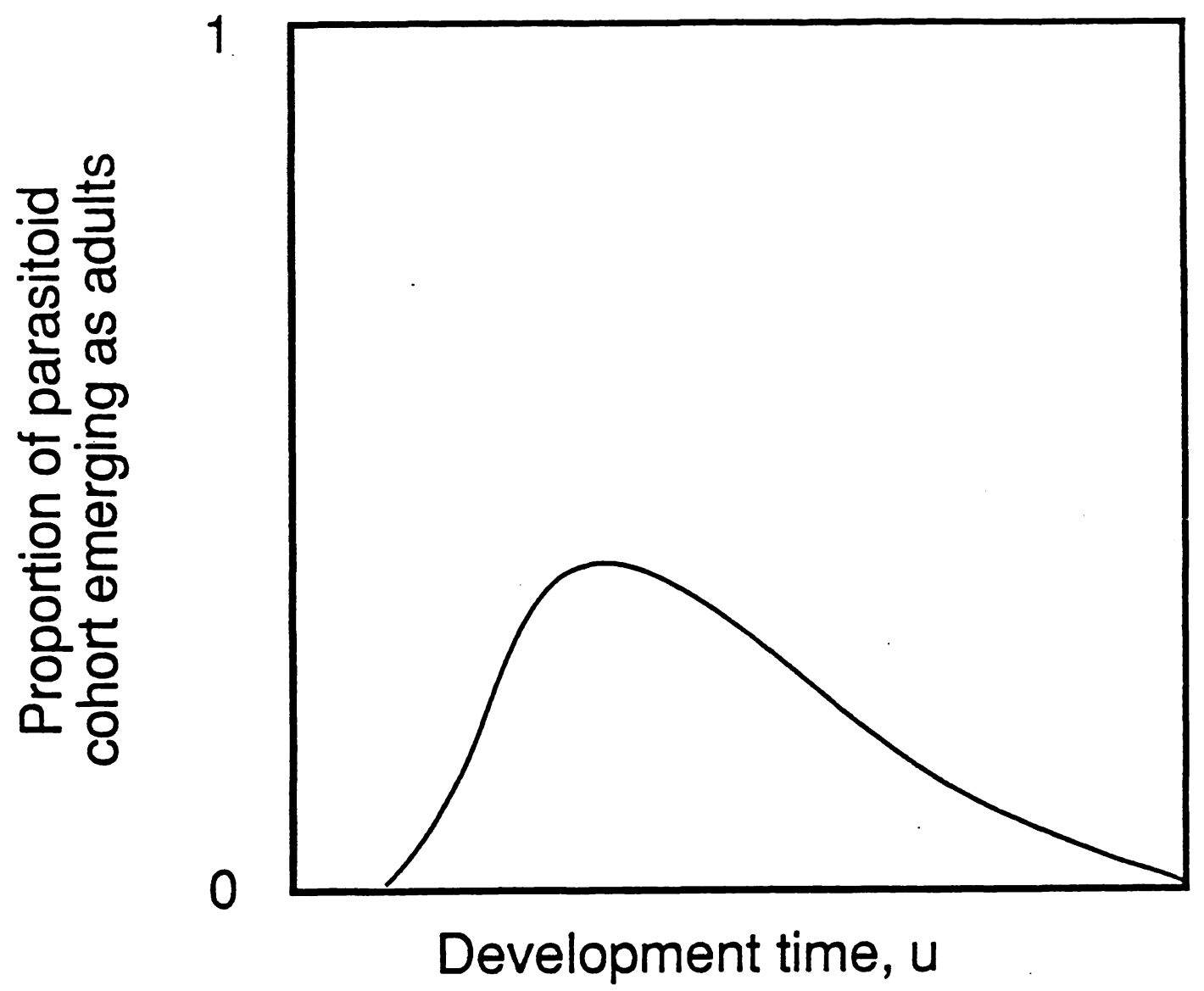

\section{Figure 2. Typical shape of adult emergence as a function of development time.}

The oviposition rate, IPS, analogous with the transmission rate in epidemiological models, fails to correspond very accurately to observed behavior (Hassell, 1986; Holling, 1959). In particular, oviposition rate per adult does not increase indefinitely with increasing availability of fly pupae, since it is limited by the number of eggs or by the handling time required for stinging and oviposition. Furthermore, this term fails to take into account the effect of interference from other adult parasitoids. This interference can take two forms: direct interference due to contact between adult parasitoids, and indirect interference due to the presence of parasitized pupae which take time to recognize as unsuitable hosts. We replace the term $I P S$ by $l P r(S, L, P)$ where $l$ is now the potential maximum oviposition rate of an adult parasitoid, and $r(S, L, P)$, the fraction of maximum rate of locating and attacking unparasitized hosts by a female parasitoid, takes on values between 0 and 1 . One expects a biologically reasonable $r$ to have the following properties: 


$$
\begin{array}{rlrl}
r(\infty, 0,0) & =1 & & \text { (Potential maximum) } \\
r(0, L, P) & =0 & & \text { (No super-parasitism) } \\
\frac{\partial r}{\partial S}>0 & & \text { (Availability of unparasitized hosts) } \\
\frac{\partial r}{\partial L} & \leq 0 & & \text { (Indirect interference) } \\
\frac{\partial r}{\partial P} \leq 0 & & \text { (Direct interference) }
\end{array}
$$

This leads to the following distributed delay model.

$$
\begin{aligned}
& S^{\prime}=\beta-l \operatorname{Pr}(S, L, P)-v S \\
& L^{\prime}=l \operatorname{Pr}(S, L, P)-\int_{0}^{\infty} l P(t-u) r(S(t-u), L(t-u), P(t-u)) p(u) e^{-\delta u} d u-\delta L \\
& P^{\prime}=\int_{0}^{\infty} l P(t-u) r(S(t-u), L(t-u), P(t-u)) p(u) e^{-\delta u} d u-n P,
\end{aligned}
$$

where the state variables are evaluated at time $t$ if not otherwise indicated, and $u$ is a dummy variable representing development time. Initial conditions for this model must specify all populations from time immemorial to the present (Bellman and Cooke, 1963). Since development times are bounded in practice, one only needs to look back as far as the oldest potential immature parasitoid.

A reasonable form for the function $r(S, L, P)$ can be derived from a simple time budget analysis (as in Arditi, 1983). We set $h_{S}$ to be the handling time for unparasitized fly pupae, $h_{L}$ to be the handling time for parasitized pupae, and $\frac{t_{0}}{S+L}$ to be time to find one pupa, assumed here for convenience to decrease linearly with increasing density of parasitized and unparasitized pupae. We assume no direct interference. Then the fraction of time spent stinging will be

$$
\frac{h_{S} \frac{S}{S+L}}{h_{S} \frac{S}{S+L}+h_{L} \frac{L}{S+L}+\frac{t_{0}}{S+L}}
$$

which can be written in the form

$$
r(S, L, P)=\frac{S}{\kappa+S+c L} .
$$

The assumptions behind this model would not be difficult to check in the laboratory, and the parameters probably not unduly difficult to estimate. 


\section{Analysis of the Model with no Direct Interference}

Throughout this section we will assume that there is no direct interference between the adult parasitoids, i.e. $\frac{\partial r}{\partial P}=0$.

If $S^{*}, L^{*}, P^{*}$ is any equilibrium of system (3), writing

$$
\begin{gathered}
\alpha=l P^{*} \frac{\partial r}{\partial S}\left(S^{*}, L^{*}\right) \\
\theta=l P^{*} \frac{\partial r}{\partial L}\left(S^{*}, L^{*}\right) \\
r^{*}=r\left(S^{*}, L^{*}\right) \\
k(z)=\int_{0}^{\infty} e^{-\delta u} e^{-z u} p(u) d u,
\end{gathered}
$$

and linearizing around the equilibrium, we obtain the characteristic equation of the system as

$$
\operatorname{det}\left[\begin{array}{ccc}
-\alpha-v-z & -\theta & -l r^{*} \\
\alpha(1-k(z)) & \theta(1-k(z))-\delta-z & l r^{*}(1-k(z)) \\
\alpha k(z) & \theta k(z) & l r^{*} k(z)-n-z
\end{array}\right]
$$

The roots of the characteristic equation determine the local stability of the equilibrium (Bellman and Cooke, 1963).

The system always has a parasitoid free equilibrium at

$$
\left(S^{*}, L^{*}, P^{*}\right)=\left(\frac{\beta}{v}, 0,0\right) \text {. }
$$

At this equilibrium $\alpha=\theta=0$, so that the roots of the characteristic equation are determined by the diagonals of the above matrix. Thus, the roots are $-v,-\delta$ and the roots of

$$
f(z) \hat{=}-n-z+l r^{*} k(z)=0 .
$$

Define $R_{0}$ to be the expected number of adult female offspring produced by an emerging adult female parasitoid when $r=1$. We may express $R_{0}$ as

$$
\begin{aligned}
R_{0} & =\text { rate of oviposition expected lifespan expected offspring survivorship } \\
& =\frac{l}{n} \int_{0}^{\infty} e^{-\delta u} p(u) d u \\
& =\frac{l}{n} k(0)
\end{aligned}
$$

At an equilibrium $\left(S^{*}, L^{*}, P^{*}\right)$, the expected lifetime offspring production $R$ of an emerging adult will be 


$$
R=R_{0} r^{*}=\frac{l}{n} k(0) r^{*}
$$

Thus we can rewrite equation (7) as

$$
-n-z+n R \frac{k(z)}{k(0)}=0 .
$$

Global stability of this equilibrium would imply the extinction of the parasitoids under any initial conditions. Since we are interested in biological control, we need to establish the conditions under which this equilibrium is unstable and the parasitoids can invade the system. We have established the following conclusion, proven in Appendix B, which is weaker than demonstrating global stability. The parasitoid free equilibrium is locally stable if and only if $R<1$. If $R>1$, equation (9) has a positive real root. Computer simulations indicate that the parasitoid-free equilibrium is globally stable if it is locally stable.

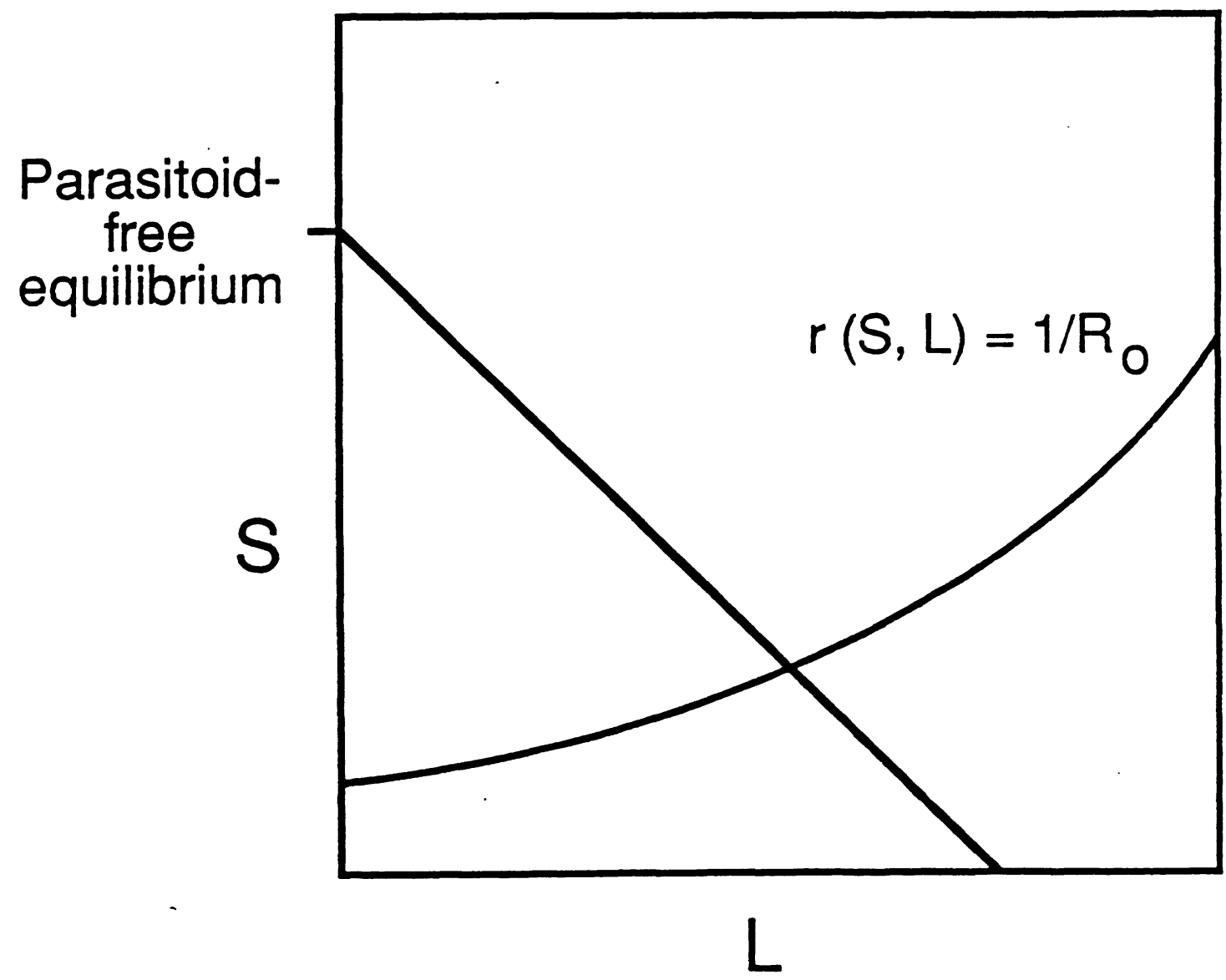

Figure 3. Equilibria of the model when there is no direct interference between adult parasitoids. See text for explanation. 
When $R$ crosses 1 , the parasitoid-free equilibrium becomes unstable by a transcritical bifurcation, producing an internal equilibrium. This equilibrium is characterized by two conditions: the first indicating that the expected number of adult offspring of an adult parasitoid must be exactly 1 , and the second relating equilibrium numbers of parasitoid larvae and fly pupae.

$$
\begin{gathered}
r^{*}=\frac{1}{R_{0}} \\
L^{*}=\frac{\beta-v S^{*}(1-k(0))}{\delta} .
\end{gathered}
$$

These conditions are sketched in figure 3. Appendix $\mathrm{C}$ provides a proof that this sketch is justified. One can see that there can be only one internal equilibrium when there is no direct interference.

At this internal equilibrium, we can write the characteristic equation as

$$
(v+z)(\delta+z)(n+z)+\alpha(\delta+z)(n+z)-\frac{n}{k(0)} k(z)(\delta+z)(v+z)-\theta(1-k(z))(n+z)(v+z)=0
$$

Evaluating this expression at $z=0$ gives $\alpha \delta n-\theta(1-k(0)) n v$ which is always greater than 0 . Therefore $z=0$ is never a root. Stability can only be lost through a Hopf bifurcation, if at all.

We wish to determine whether predator-prey type oscillations are possible around this equilibrium. We have only been able to successfully analyze the case with no indirect interference, that is $\theta=0$. In this case, where adult parasitoids essentially do not interact, we show in Appendix D that the internal equilibrium is always locally stable.

This is not the most interesting case biologically, since it assumes that adult parasitoids do not waste any time or energy in recognizing parasitized fly pupae or in fighting with each other. Computer simulations indicate that stability is preserved in the case of indirect interference, and a computer algebra proof using MACSYMA has been produced for $p(u)$ of the form $m^{2} e^{-m u}$ for an arbitrary biologically reasonable function $r(S, L)$. We conjecture that this stability property holds for arbitrary unimodal $p(u)$.

As shown in Nisbet and Gurney (1976), strong direct interference producing overcompensation is almost certain to produce oscillations. Further experments are needed to determine whether this is a possibility, and what the mechanism for such interference might be.

\section{Effects of parasitoid parameters on internal equilibrium}

In Nicholson-Bailey style models (see Beddington et al., 1978), the internal equilibrium number of hosts cannot be driven to zero with increasing predator efficiency. In our model, for some forms of the function $r$, the equilibrium value $S^{*}$ can be arbitrarily small. Of course, much of the difference here concerns our assumptions about the dynamics of the fly population; no matter how low the pupal population becomes, there is still a constant supply of fresh pupae.

Using the handling time derivation of $r$ as in equation (4), condition (9a) for an equilibrium becomes 


$$
S\left(R_{0}-1\right)=\kappa+c L,
$$

where $\kappa$ represents a measure of searching efficiency and $c$ is the ratio of handling times of parasitized to unparasitized fly pupae. We can thus solve for $S^{*}$ as

$$
S^{*}=\frac{\kappa+\beta c \Delta}{\left(R_{0}-1\right)+v c \Delta}
$$

where $\Delta=\frac{1-k(0)}{\delta} . \Delta$ has a biological interpretation as the mean amount of time spent in the larval stage, at least in the case where $q$ (the fraction of larvae which mature but fail to emerge) is equal to 0. This is shown in Appendix E.

We also know that the existence of an internal equilibrium requires $R_{0} r\left(\frac{\beta}{v}, 0,0\right)>1$, or with this $r, R_{0}>1+\frac{\kappa V}{\beta}$. We can therefore write

$$
R_{0}=1+\zeta \frac{\kappa v}{\beta}
$$

for $\zeta>1$. Then

$$
S^{*}=\frac{\beta}{v}\left[\frac{\kappa+\beta c \Delta}{\kappa \zeta+\beta c \Delta}\right]
$$

We can thus see immediately that if $R_{0}$ increases due to changes in the adult parameters $l$ and $n$, that the equilibrium level of pupae decreases. Also, $S^{*}$ increases monotonically with increasing larval residence time, as expected, since this indicates a slower rate of natural increase for the parasitoid population. This result exposes another weakness of the model assumptions, in that fly pupae only affect adult parasitoid searching behavior while they contain live fly or parasitoid larvae. This is probably not the case for parasitoids of house fly pupae. We expect experiments to indicate that unoccupied or dead fly pupae have a decreasing interference effect as they age. This component could be incorporated into the model by addition of a class of dead fly pupae.

\section{CONCLUSIONS AND FURTHER DIRECTIONS}

The analysis of the distributed-delay model developed in this paper indicates several ideas regarding biological control. A host-parasitoid model in which the recruitment of susceptible hosts is unaffected by local dynamics, has a tendency to converge to a stable equilibrium whether or not the parasitoids can persist. This equilibrium can have arbitrarily small numbers of susceptible hosts when the parasitoids are extraordinarily efficient. We show that the ability of the parasitoids to persist can be established in terms of basic fly and parasitoid life history parameters.

Even without including the difficulties associated with dispersal and foraging behavior, several modifications may be necessary to use this model in the field. Adult parasitoids sting fly pupae, without laying eggs, in order to drink the hemolymph (Patterson and Rutz, 1986). This direct predation could be easily incorporated into the model if measurements indicate that it takes place at a rate 
roughly proportional to the rate of oviposition. Super-parasitism occurs in many species of parasitoid, especially when unparasitized hosts are scarce. Tests need to be made of this potentially complicating factor. The sex ratios of parasitoid progeny can vary in response to host abundance and quality, interference, and environmental conditions (Waage, 1986). This could invalidate the total population number approach taken in this model. Also, as noted above, after a fly or parasitoid emerges from a fly pupa, the puparium remains in the environment and may be confusing to foraging parasitoids. Measures of the indirect interference component of the model need to incorporate this effect.

Two plausible and tractable modifications could be incorporated in the model to make the host dynamics more realistic. One could make the local recruitment rate $\beta$ a function of temperature or time, perhaps fitting it to measured abundance throughout the summer breeding season. Alternatively, by modelling as a closed system, one could estimate the ability of parasitoids to control hosts below their environmental carrying capacity (Andreasen, ms.). Most simply, one could set the local fly recruitment rate to be $\beta S$, which assumes that the fly life cycle is significantly shorter than that of the parasitoid.

Experimental tests will show whether this model framework warrants extension to more realistic situations.

\section{Acknowledgments}

F. Adler and C. Castillo-Chavez acknowledge the support of McIntire-Stennis grant (NYC183568) and the National Science Foundation grant DMS-8406472 to S. A. Levin. C. CastilloChavez acknowledges also the support of the Office of the Provost, the Center for Applied Mathematics at Cornell University, and a Ford Foundation Postdoctoral fellowship to minorities. 


\section{Appendix A}

We wish to show that the system of delay differential equations (A1)

$$
\begin{gathered}
S^{\prime}=\beta-l P S-v S \\
L^{\prime}=l P S-m I-\delta L \\
P^{\prime}=m I-n P \\
I=\int_{0}^{\infty} l P(t-u) S(t-u) p(u) e^{-\delta u} e^{-m u} d u
\end{gathered}
$$

has solutions coinciding with those of the system of ordinary differential equations (A2) under suitable initial conditions

$$
\begin{gathered}
S^{\prime}=\beta-l P S-v S \\
L^{\prime}=l P S-m L-\delta L \\
P^{\prime}=m L-n P .
\end{gathered}
$$

Suppose the initial conditions of (A1) satisfy (A2) on $(\infty, 0)$. Then

$$
I^{\prime}=-(m+\delta) I+l P S .
$$

Hence if $I(0)=L(0)$, we have that $I(t)=L(t)$ for all times $t>0$.

\section{Appendix B (Proof of threshold result)}

The parasitoid free equilibrium is locally stable if and only if $R<1$. If $R>1$, equation (8) has a positive real root.

Suppose $R<1$ and $z=a+\omega i$ is a root of (8) with $a>0$. We have that

$$
\left|\operatorname{Re}(k(i \omega))=\int_{0}^{\infty} e^{-a u} \cos (\omega u) e^{-\delta u} p(u) d u\right|<k(0),
$$

so the real part of equation (8) satisfies

$$
-n-a+n R \frac{\operatorname{Re}(k(i \omega))}{k(0)}<-n+n R<0 .
$$

Therefore all characteristic roots lie in the negative half plane when $R<1$. It is obvious that $z=0$ is a root when (and only when) $R=1$. By the implicit function theorem, there is a real solution $z(R)$ for $R$ near 1 if

$$
\frac{\partial}{\partial z}\left[-n-z+n R \frac{k(z)}{k(0)}\right] \neq 0 .
$$


But for real $z$ we have that

$$
\frac{\partial f}{\partial z}=-1-\frac{n R}{k(0)} \int_{0}^{\infty} u e^{-z u} p(u) d u<0
$$

and

$$
\frac{\partial f}{\partial R}=n \frac{k(z)}{k(0)}>0
$$

Therefore

$$
\frac{d z}{d R}=-\frac{\frac{\partial f}{\partial R}}{\frac{\partial f}{\partial z}}>0
$$

Thus there is always a real root, which is positive for $R>1$.

\section{Appendix C (Uniqueness of internal equilibrium)}

We have that $r\left(\frac{\beta}{v}, 0\right)>\frac{1}{R_{0}}$, hence $r\left(S_{0}, 0\right)=\frac{1}{R_{0}}$, for some unique $S_{0}<\frac{\beta}{v}$. By the implicit function theorem, the equation $r(S(L), L)=\frac{1}{R_{0}}$ will have a solution for the function $S(L)$ if $\frac{\partial r}{\partial S} \neq 0$, which is assumed. The conditions (5) imply that $\frac{d S}{d L}>0$. This shows that figure 3 is correct and that there is necessarily a unique intersection.

\section{Appendix D (Stability in no interference case)}

Equation (10) can be factored as

$$
(\delta+z)\left[(v+z)(n+z)+\alpha(n+z)-\frac{n}{k(0)} k(z)(v+z)\right]=(\delta+z) g(z)
$$

Evaluating $g(z)$ at $z=i \omega$, we get

$$
\begin{aligned}
k(i \omega) & =\left[\int_{0}^{\infty} e^{-\delta u} \cos (\omega u) p(u) d u\right]-i\left[\int_{0}^{\infty} e^{-\delta u} \sin (\omega u) p(u) d u\right] \\
& =k_{R}(\omega)-i k_{i}(\omega)
\end{aligned}
$$

The real and imaginary parts of $g(i \omega)$ are

$$
n v-\omega^{2}+\alpha n-n v \frac{k_{R}(\omega)}{k(0)}-n \omega \frac{k_{I}(\omega)}{k(0)}=0
$$




$$
\omega(n+v+\alpha)+n v \frac{k_{I}(\omega)}{k(0)}-n \omega \frac{k_{R}(\omega)}{k(0)}=0
$$

Multiplying the first equation by $v$ and the second by $\omega$ and adding gives

$$
\alpha\left(n v+\omega^{2}\right)+n\left(v^{2}+\omega^{2}\right)\left[1-\frac{k_{R}(\omega)}{k(0)}\right]=0
$$

But $k_{R}(\omega) \leq k(0)$, hence there is no solution and no Hopf bifurcation in this case.

\section{Appendix $\mathbf{E}$ (The interpretation of $\Delta$ )}

If $p(u)$ is $\delta \tau(u)$, the point density at $\tau$ corresponding to constant development time and a discrete delay, the density of disappearance times from the larval stage due to either death or maturation is

$$
\delta e^{-\delta u} I_{[0, \tau]}+\left(\int_{\tau}^{\infty} \delta e^{-\delta s} d s\right) \delta \tau(u),
$$

where $I$ represents the indicator function of the given interval. Evaluating the mean of this density gives

$$
\frac{1-e^{-\tau}}{\delta}
$$

For a general $p(u)$ then, the mean time in the larval state will be

$$
\int_{0}^{\infty} \frac{1-e^{-\tau}}{\delta} p(\tau) d \tau=\frac{1-k(0)}{\delta} .
$$




\section{BIBLIOGRAPHY}

Anderson, R. M. and R. M. May, "The population dynamics of microparasites and their invertebrate hosts," Phil. Trans. Roy. Soc. B, vol. 291, pp. 451-524, 1981 .

Andreasen, V., Disease regulation of age-structured host populations. In press

Arditi, R., "A unified model of the functional response of predators and parasitoids," J. Anim. Ecol., vol. 52, pp. 293-303, 1983 .

Beddington, J. R., C. A. Free, and J. H. Lawton, "Characteristics of successful natural enemies in models of biological control of insect pests," Nature, vol. 273, pp. 513-519, 1978.

Bellman, R. and K. Cooke, Differential-difference equations, Academic Press, New York, 1963.

Caltagirone, L. E., "Landmark examples in classical biological control," Ann. Rev. Entomol., vol. 26, pp. 213-232, 1981.

Danthanarayana, W., Insect flight - dispersal and migration, Springer-Verlag, New York, 1986.

Greathead, D., "Parasitoids in Classical Biological Control," in Insect Parasitoids, ed. J. Waage and D. Greathead, pp. 290315, Academic Press, New York, 1986.

Hassell, M. P. and R. M. May, "Stability in insect host-parasite models," J. Anim. Ecol., vol. 42, pp. 693-736, 1973.

Hassel1, M. P., The Dynamics of Arthropod Predator-Prey Systems, Princeton University Press, Princeton, 1978.

Hassell, M. P., "Foraging strategies, population models and biological control: A case study," J. Anim. Ecol., vol. 49, pp. $603-628,1980$.

Hassell, M. P., "Parasitoids and Population Regulation," in Insect Parasitoids, ed. J. Waage and D. Greathead, pp. 201222, Academic Press, New York, 1986.

Holling, C. S., "Some characteristics of simple types of predation and parasitism," Can. Ent., vol. 91, pp. 385-398, 1959. 
Huffaker, C. B. and P. S. Messenger, Theory and Practice of Biological Control, Academic Press, New York, $\overline{1976 .}$

May, R. M., "Host-parasitoid systems in patchy environments: a phenomonological model," J. Anim. Ecol., vol. 47, pp. 833$843,1978$.

May, R. M., M. P. Hassell, R. M. Anderson, and D. W. Tonkyer, "Density dependence in host-parasitoid models," J. Anim. Ecol., vol. 50, pp. 855-865, 1981.

Murdoch, W.W., J. D. Reeve, C. B. Huffaker, and C. E. Kennett, "Biological control of olive scale and its relevance to ecological theory," Am. Nat., vol. 123, pp. 371-392, 1984.

Murdoch, W. W., J. Chesson, and P. I. Chesson, "Biological control in theory and practice," Am. Nat., vol. 125, pp. 344366,1985 .

Murdoch, W. W., R. M. Nisbet, S. Blythe, R. Gurney, and J. D. Reeve, "An invulnerable age class and stability in delaydifferential parasitoid-host models," Am. Nat., vol. 129, pp. 263-282, 1987 .

Nisbet, R. M. and W. S. G. Gurney, "A simple mechanism for population cycles," Nature, vol. 263, pp. 319-320, 1976.

Patterson, R. S. and D. A. Rutz, Biological control of muscoid flies, Entomol. Soc. Amer., misc. pub. $61,1986$.

Reeve, J.D. and W.W. Murdoch, "Aggregation by parasitoids - the successful control of the California Red Scale: A test of theory," J. Anim. Ecol., vol. 54, pp. 797-816, 1985.

Ridgway, R. I. and S. B. Vinson, Biological control by augmentation of natural enemies: insect and mite control with parasites and predators, $\overline{\text { Plenum }} \overline{\text { Press, New York, }} \overline{1977 .}$

Smith, L. and D. A. Rutz, "The occurence an biology of Urolepis rufipes (Hymenoptera: Pteromalida), a parasitoid of house flies in New York dairies," Environ. Entomol., vol. 14, pp. $365-369,1985$.

Smith, L. and D. A. Rutz, "Devolopment rate adn survivorship of immature Urolepis rufipes (Hymenoptera: Pterolmalidae), a parasitoid of pupal house flies.," Environ. Entomol., vol. 15, pp. 1301-1306, 1986.

Smith, L. and D. A. Rutz, "Reproduction, adult survival and intrinsic rate of growth of Urolepis rufipes (Hymenoptera: Pteromalidae), a pupal parasitoid of house flies, Musca domestica," Entomophaga, vol. 32, In press. 
Waage, J. and D. Greathead, Insect Parasitoids, Academic Press, London, 1986.

Waage, J. K., "Family Planning in Parasitoids: Adaptive Patterns of Progeny and Sex Allocation," in Insect Parasitoids, ed. J. Waage and D. Greathead, pp. 201-222, Academic Press, New York, 1986. 